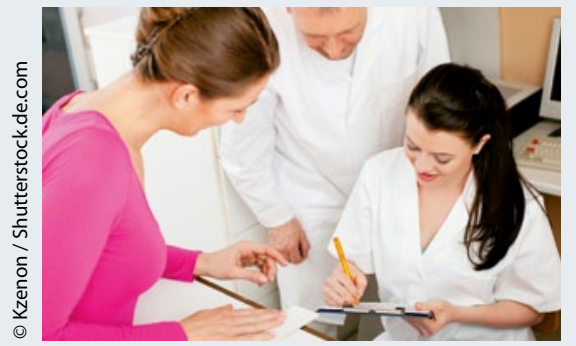

Das verdienen junge MFA

Medizinische Fachangestellte (MFA) werden nach der Ausbildung in Hamburg, Hessen und Bayern am besten bezahlt. Dabei winkt den Berufseinsteigerinnen in Hessen mit im Schnitt $22.093 €$ das höchste Jahresgehalt. In Hamburg bekommen sie immerhin noch $20.769 €$ und in Bayern von 20.399 €. Das zeigt eine Studie des Internet-portalbetreibers PersonalMarkt Services $\mathrm{GmbH}$, bei der die Gehaltsdaten von 5.526 MFA aus neun Bundesländern ausgewertet wurden. Das niedrigste Einstiegsgehalt gibt es demnach mit $17.942 €$ in Brandenburg.

\section{Gesundheitsbranche bleibt Jobmotor}

Für 2012 zeichnet sich in der Gesundheitsbranche ein Zuwachs von 70.000 neuen Stellen ab. Das geht aus einer Umfrage der Deutschen Industrie- und Handelskammer hervor, die 1.000 Unternehmen dieser Branche befragt hatte. Das größte Risiko für die wirtschaftliche Entwicklung ist für $42 \%$ der befragten Unternehmen der Fachkräftemangel. Mit $68 \%$ fürchten vor allem Pflegedienste, angesichts des steigenden Pflegebedarfs nicht genügend qualifiziertes Personal zu finden. In der Pharmabranche sehen 32\% im Fachkräftemangel ein großes Risiko, ähnlich wie die Medizintechnik (30\%).

\section{PKV will Spezialtarif für Nichtzahler}

Säumige Beitragszahler verhageln der PKV die Bilanzen - und die Zahl der Schuldner wächst offenbar. Die Versicherer verhandeln nun über eine Lösung: ein Spezialtarif für Nichtzahler, der allerdings nur Notfälle abdecken würde. Zurzeit verhandelt der PKV-Verband darüber mit verschiedenen Bundesministerien. Branchenweit gab es Ende Juni 2011 142.800 Nichtzahler. Nach Informationen der Debeka beliefen sich die durch sie ausgelösten Verluste am Ende des dritten Quartals auf 554 Millionen Euro. Seit Einführung der Versicherungspflicht können die Unternehmen Kunden, die ihre Beiträge schuldig bleiben, nicht mehr kündigen.

\section{BÄK strebt eine GOÄ-Novelle für 2013 an}

\author{
Die Bundesärztekammer (BÄK) geht nicht mehr davon aus, dass eine Novelle \\ der GOÄ im Jahr 2012 unter Dach und Fach kommt. Ziel ist nun, eine Reform \\ der GOÄ noch vor der Bundestagswahl 2013 zu schaffen.
}

$\mathrm{D}$ ie Ärzte gehen mit einem betriebswirtschaftlich neu durchgerechneten Leistungskatalog in die Verhandlungen über eine neue GOÄ, hat die stellvertretende Hauptgeschäftsführerin der BÄK; Dr. Regina Klakow-Franck; auf Anfrage gesagt. Ziel sei es, die neu kalkulierten Leistungen mit „robusten Einfachsätzen“ in Euro auszustatten. Über diese Einfachsätze soll auch die Kostenentwicklung in den Arztpraxen seit der letzten GOÄ-Novelle abgebildet werden.

Die Kammer sei gut auf die Verhandlungen mit den Kostenträgern und der Bundesregierung vorbereitet, die nach der Verabschiedung der Gebührenordnung für Zahnärzte jetzt anstehen. Die Materie sei aber sehr komplex. „Wenn sich die verschiedenen Beteiligten im Vorfeld einigen können und die Vorarbeiten der BÄK als
Ausgangsbasis nehmen, dann könnte man es vor der Bundestagswahl schaffen", so die Einschätzung Klakow-Francks, die auch Leiterin des Dezernats Gebührenordnung bei der BÄK ist.

Durch die GOÄ-Novelle soll es nach der Vorgabe von Dr. Theodor Windhorst aus dem Vorstand der BÄK eine „Zweistellige Erhöhung" der Honorare geben. Die Zahnärzte hatten rechnerisch über die GOZNovelle ein Plus von $6 \%$ erreicht. Bundesgesundheitsminister Daniel Bahr (FDP) ermahnte die Ärzte derweil zu Kompromissbereitschaft.

Ein weiteres Ziel sei für die BÄK eine Vereinfachung der Abrechnung, sagte Klakow-Franck. Es werde zwar mehr Positionen in der GOÄ geben, aber pro Fall sollten am Ende weniger Ziffern auf einer Abrechnung stehen.

\section{Abrechnungstipp!}

\section{Als Konsiliarius im Krankenhaus}

- Da nicht jedes Krankenhaus über eine neurologische Abteilung ver-

fügt, kommt es nicht selten vor, dass gerade Neurologen zur fachärztlichen Konsiliartätigkeit auch bei GKV-Patienten im Auftrag einer Klinik kommen. Weil dabei die Honorarmodalitäten immer wieder zu Diskrepanzen auch juristischer Art führen können, hat der BGH in 2009 ein Urteil gefällt, dass Vereinbarungen zwischen Vertragsärzten und Krankenhäusern in diesen Fällen nicht den Vorschriften der GOÄ unterliegen (Aktenzeichen III ZR 110/09). Mit anderen Worten: Hier kann es durchaus zur vertraglichen Vereinbarung von Pauschalen kommen. Gleichzeitig besteht allerdings auch die Möglichkeit der vereinbarten Abrechnung nach GOÄ, wobei es dann von der Kooperationswilligkeit beider Partner und der "Marktmacht" beider Partner abhängt, wie der Steigerungsfaktor geregelt wird.

Anders gelagert ist die Situation in der Regel bei Selbstzahlerpatienten im Krankenhaus. Hier hat der Konsiliararzt die Möglichkeit, direkt mit dem Patienten selbst abzurechnen. In diesen Fällen gelten dann automatisch die Vorgaben der GOÄ mit allen Rechten und Pflichten. Denn nach dem Krankenhausentgeltgesetz ist der Auftraggeber des Konsiliararztes nicht das Krankenhaus, sondern der Patient selbst, auch wenn normalerweise der Konsiliararzt durch einen Mitarbeiter des Krankenhauses gerufen wird. Allerdings muss auch in diesen Fällen die Rechnung bei stationär behandelten Patienten um 15\% gemindert werden, wenn die Leistung von einem niedergelassenen Arzt erbracht wird (GOÄ § 6a, Abs. 1). Fazit: Bei Konsiliartätigkeit am Krankenhaus sollte grundsätzlich auf unterschiedliche Honorarmöglichkeiten von GKV-Patienten und Privatpatienten geachtet werden

Dr. med. Heiner Pasch 